\title{
Environmental limits on the non-resonant cosmic-ray current-driven instability
}

\author{
B. Reville, J.G. Kirk \\ Max-Planck-Institut für Kernphysik, Heidelberg 69029 \\ P. Duffy \\ UCD School of Physics, University College Dublin, Belfield, Dublin 4 \\ S. O'Sullivan \\ School of Mathematical Sciences, Dublin City University, Glasnevin, Dublin 9
}

\begin{abstract}
We investigate the so-called non-resonant cosmic-ray streaming instability, first discussed by Bell (2004). The extent to which thermal damping and ion-neutral collisions reduce the growth of this instability is calculated. Limits on the growth of the non-resonant mode in SN1006 and RX J1713.7-3946 are presented.
\end{abstract}

Keywords: cosmic rays; magnetic fields

\section{Introduction}

Diffusive shock acceleration at the outer shocks of supernova remnants is believed to be the primary source for galactic cosmic rays. In the simplest picture, however, the maximum energy attainable by this mechanism falls short of the so-called knee at approx. $1 \mathrm{PeV}$ [1]. Amplification of the magnetic field in the vicinity of shock front may facilitate acceleration to higher energies, without changing the simplicity of the model. There has also been an increase in the wealth of observational evidence for magnetic field amplification at collisionless shocks, such as the non-thermal bright X-ray rims in several supernova remnants $[2,3]$. The growth of the magnetic field is thought to be closely related to the efficient production of cosmic rays and the non-resonant current-driven instability [4] has provided a mechanism which may indeed considerably amplify the magnetic field.

Generally speaking, the medium upstream of a supernova blastwave will not be completely ionised. The presence of a small neutral component limits the acceleration of cosmic rays in these environments [5], due to damping of resonantly excited Alfvén waves. In molecular clouds, where the ionisation fractions are even lower and the densities much higher, the situation is even more extreme $[6,7]$. In a certain range of wavelengths, Alfvén waves simply do not propagate, and are quite rapidly damped.

A non-resonant mode, driven by a cosmic-ray current in a partially ionised medium has recently been studied [8]. Treating the background particles as a MHD single fluid with a generalised Ohm's law, and using a kinetic description of the cosmic rays, these authors conclude that a non-resonant mode with a rapid growth rate remains. However, as we show, for high frequency waves the friction between the charged species and the neutrals is not sufficient to couple their motions, and a single fluid description of the plasma is no longer appropriate.

In the present work, we investigate various conditions that may reduce the growth of the non-resonant instability. We first calculate the effect of thermal damping of the waves. We also determine an expression for the dispersion relation of waves in the presence of an arbitrary fraction of neutral particles. These results are then used to speculate on whether or not magnetic field amplification may have taken place in SN1006 or in the molecular cloud close to RX J1713.7-3946. We conclude with some remarks about the importance of the non-resonant currentdriven instability.

\section{Thermal effects}

We present here a simple derivation of the cosmic-ray current-driven instability. Since the fastest growing mode is non-resonant we do not need to calculate the full effects of an anisotropic cosmic-ray distribution [9], and we investigate here a super-Alfénic beam, which considerably simplifies the analysis.

The linear dispersion relation for circularly polarised transverse waves propagating parallel to the 
zeroth order magnetic field is

$$
\frac{c^{2} k^{2}}{\omega^{2}}-1=\sum_{s} \chi_{s}(k, \omega)
$$

where the summation is over each species with corresponding charge $q_{s}$, cyclotron frequency $\omega_{\mathrm{cs}}=$ $q_{s} B_{0} / m_{s} c$ and plasma frequency $\omega_{\mathrm{ps}}^{2}=4 \pi q_{s}^{2} n_{s} / m_{s}$. The susceptibility $\chi_{s}$ for each component of a plasma is determined by integrating the Vlasov equation along the unperturbed trajectories about the zeroth order field and can be found in any standard textbook on plasma physics (e.g. Krall \& Trivelpiece 1973). For circularly polarised waves propagating parallel to a uniform magnetic field, the susceptibility for each species is given by

$$
\begin{aligned}
\chi_{s}=\frac{4 \pi q_{s}^{2}}{\omega^{2}} \int \mathrm{d}^{3} p \frac{v_{\perp} p_{\perp}}{\omega \pm \omega_{\mathrm{cs}}-k v_{\|}} \\
{\left[\left(\omega-k v_{\|}\right) \frac{\partial f_{s}}{\partial p_{\perp}^{2}}+k v_{\|} \frac{\partial f_{s}}{\partial p_{\|}^{2}}\right] . }
\end{aligned}
$$

For simplicity, the plasma we consider has only three components. A two species thermal population of electrons and protons, and a cosmic ray component which we assume to consist entirely of protons. Since we are investigating waves on lengthscales longer than the Debye length the plasma can be taken to be quasi-neutral, resulting in a slight charge excess of electrons in the thermal plasma due to the presence of cosmic rays. A neutralising background flux is also induced to balance the current produced by the streaming cosmic rays.

Assuming a Maxwellian background and a mono-energetic beam of protons with momentum $p_{0}$ directed along the magnetic field, it is straightforward to show that the dispersion relation for low frequency waves $|\omega| \ll \omega_{\text {ci }}<\left|\omega_{\text {ce }}\right|$ is

$$
\frac{\omega^{2}}{k^{2} c^{2}}+\epsilon \frac{\omega}{\omega_{\mathrm{ci}}} \Theta-\frac{\mathrm{v}_{\mathrm{A}}^{2}}{c^{2}}-\frac{\zeta \beta_{\mathrm{b}}^{2}}{1-\epsilon k r_{\mathrm{g}}}=0
$$

where $c \beta_{\mathrm{b}}$ is the speed of the proton beam, $\mathrm{v}_{\mathrm{A}}=$ $B_{0} / \sqrt{4 \pi n_{\mathrm{i}} m_{\mathrm{i}}}$ is the Alfvén velocity and $\Theta=$ $k_{B} T_{\mathrm{i}} / m_{\mathrm{i}} c^{2}$ the dimensionless ion temperature. We have also introduced the parameter $\epsilon$ to describe the polarisation with $\epsilon=+1(-1)$ for right (left)-handed waves $(\omega>0)$.

We define $r_{\mathrm{g}}=p_{0} c / e B_{0}$ the gyroradius of the protons in the beam and consider only waves with $k>0$. We have also introduced a dimensionless parameter characterising the strength of the driving term

$$
\zeta=\frac{n_{\mathrm{cr}} p_{0}}{n_{\mathrm{i}} m_{\mathrm{i}} \beta_{\mathrm{b}} c}
$$

The maximum growth rate of the non-resonant mode is

$$
\operatorname{Im}(\omega) \approx \frac{1}{2} \frac{c}{\mathrm{v}_{\mathrm{A}}} \frac{n_{\mathrm{cr}}}{n_{\mathrm{i}}} \beta_{\mathrm{b}} \omega_{\mathrm{ci}},
$$

which is independent of magnetic field strength.

We can now determine possible thermal effects on the growth rate. For $k r_{\mathrm{g}} \gg 1$, it follows from (3), in the limit $\Theta \gg \mathrm{vA}^{2} / c^{2}$, that the maximum growth rate is

$$
\operatorname{Im}(\omega) \sim\left(n_{\mathrm{cr}} / n_{\mathrm{i}}\right)^{2 / 3}\left(\beta_{\mathrm{b}}^{2} / \Theta\right)^{1 / 3} \omega_{\mathrm{ci}}
$$

In order for the non-resonant mode to leave the regime of linear growth before being overtaken by the shock front, i.e., before being advected over a distance of roughly $r_{\mathrm{g}} / \beta_{\mathrm{b}}$, one requires $\operatorname{Im}(\omega)>\beta_{\mathrm{b}}^{2} c / r_{\mathrm{g}}$. The necessary condition for thermal effects to reduce the growth rate below this value is

$$
\Theta>\frac{\zeta^{2} p_{0}}{\beta_{\mathrm{b}}^{2} m_{\mathrm{i}} c} .
$$

For typical SNR parameters this condition will only be satisfied for a very weak driving term , $\zeta \ll 1$. However, thermal effects are likely to play a significant role for relativistic shocks, and may even provide a saturation mechanism for the current driven instability [10].

\section{Collisional effects}

The surroundings into which supernova shocks propagate are, in general, not completely ionised. Observations have shown that many supernova take place near molecular clouds, which can have very low ionisation fraction. We consider the reduction of the growth of the non-resonant modes due to ion-neutral friction. For wave frequencies larger than the momentum exchange frequency, a multi-fluid treatment of the plasma is necessary, since the collisions are not frequent enough to couple the motion of the neutral particles to the charged ions. We consider two fluids, a single MHD fluid for the charged species and a neutral component. A simple analysis of the frictionally coupled system with an external cosmic ray current results in the following dispersion relation [9]

$$
\omega^{2}\left(1+\frac{i \nu_{\mathrm{in}}}{\omega+i \nu_{\mathrm{ni}}}\right)=k^{2} \mathrm{v}_{\mathrm{A}}^{2}+\epsilon \zeta \frac{\mathrm{v}_{\mathrm{s}}^{2}}{r_{\mathrm{g}}} k(\sigma-1) .
$$


where $\nu_{a b}$ is the momentum exchange frequency from species $a$ to species $b$ and are related by momentum conservation $\rho_{\mathrm{n}} \nu_{\mathrm{ni}}=\rho_{\mathrm{i}} \nu_{\mathrm{in}}$. The complex function $\sigma(k)$ represents the susceptibility of the cosmic rays normalised to the induced thermal return current $[4,10]$. For plasmas in the temperature range $10^{2} \mathrm{~K}<T<10^{5} \mathrm{~K}$, [11] give the following expression

$$
\nu_{\text {in }} \approx 8.9 \times 10^{-9} n_{\mathrm{n}}\left(\frac{T}{10^{4} \mathrm{~K}}\right)^{0.4} \mathrm{~s}^{-1} .
$$

In the high frequency limit, $|\omega| \gg \nu_{\text {in }}$, this reduces to a dispersion relation similar to Eq. (3). In the low frequency limit $|\omega| \ll \nu_{\text {ni }}$ we find

$$
\omega^{2} \approx \frac{\rho_{\mathrm{i}}}{\rho}\left[k^{2} \mathrm{v}_{\mathrm{A}}^{2}+\epsilon \zeta \frac{\mathrm{v}_{\mathrm{s}}^{2}}{r_{\mathrm{g}}} k(\sigma-1)\right] .
$$

If the collision frequencies are very large, the neutral and ionised components are tied together and the effect of ion-neutral collisions is simply to increase the effective mass of the ions.

For strongly driven, non-resonant modes Eq. (7) reduces to

$$
\omega\left(\omega^{2}+\zeta \frac{\mathrm{v}_{\mathrm{s}}^{2}}{r_{\mathrm{g}}} k\right)+i \nu_{\text {in }}\left[(1+Z) \omega^{2}+Z \zeta \frac{\mathrm{v}_{\mathrm{s}}^{2}}{r_{\mathrm{g}}} k\right]=0,
$$

where $Z=\rho_{\mathrm{i}} / \rho_{\mathrm{n}}=\nu_{\mathrm{ni}} / \nu_{\mathrm{in}}$. For all physically relevant parameters, this cubic polynomial in $\omega$ has three purely imaginary roots, two of which are damped modes, and the third the non-resonant growing mode. Thus, ion-neutral collisions are unable to stabilise the strongly driven mode, although they can affect its growth rate. Interestingly this result also applies to any aperiodic mode.

The general analytic expressions for the growing mode are cumbersome, but various limiting cases yield interesting results. In the limit of low ionisation, $Z \gg 1$ one recovers the growth rate given in Eq. (4). For $Z \ll 1$ the growth rate is given by

$$
\gamma=-\frac{\nu_{\text {in }}}{2}+\frac{1}{2} \sqrt{\nu_{\text {in }}^{2}+4 \zeta \frac{\mathrm{v}_{\mathrm{s}}^{2}}{r_{\mathrm{g}}} k}
$$

If $\nu_{\text {in }}^{2} \ll 4 \zeta \mathrm{v}_{\mathrm{s}}^{2} k / r_{\mathrm{g}}$ collisions are too slow to compete with the driving, and the growth rate is again the same as Eq. (4) with a negligible reduction due to collisions. However, in addition to the low frequency limit in which the components are tied together Eq. (9), a new regime arises. If the collisional drag on the ions is sufficient to affect the driving, but not to couple the components, i.e., $\nu_{\mathrm{in}}^{2} \gg 4 \zeta \mathrm{v}_{\mathrm{s}}^{2} k / r_{\mathrm{g}} \gg \nu_{\mathrm{ni}}^{2}$, then we obtain

$$
\gamma \approx \zeta \frac{\mathrm{v}_{\mathrm{s}}^{2}}{\nu_{\mathrm{in}} r_{\mathrm{g}}} k=\frac{\omega_{\mathrm{ci}}}{\nu_{\mathrm{in}}} \frac{n_{\mathrm{cr}}}{n_{\mathrm{i}}} k \mathrm{v}_{\mathrm{s}} .
$$

A similar result to this has been found previously [8], but as we have shown here, this is valid only when $\gamma \ll \nu_{\text {in }}$.

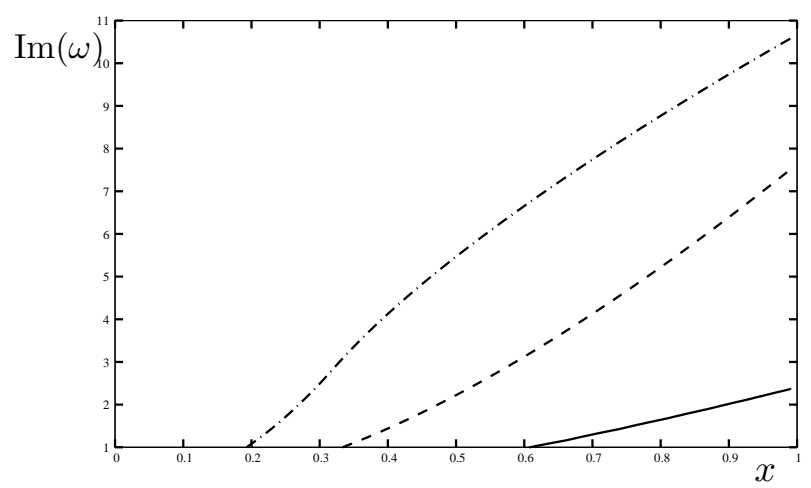

Fig. 1. Maximum growth rate as a function of ionisation fraction for total density $n=n_{\mathrm{i}}+n_{n}$ in a $\mathrm{H}-\mathrm{H}^{+}$gas: $0.1 \mathrm{~cm}^{-3}, 10^{4} \mathrm{~K}$ (solid), $1.0 \mathrm{~cm}^{-3}, 10^{3} \mathrm{~K}$ (dash), $10.0 \mathrm{~cm}^{-3}$, $10^{2} \mathrm{~K}$ (dash-dot). We take $\zeta=0.01 x_{\mathrm{i}} \mathrm{v}_{\mathrm{s}} / c$ with shock speed $\mathrm{v}_{\mathrm{s}}=5000 \mathrm{~km} \mathrm{~s}^{-1}$, and $r_{\mathrm{g}}=10^{14} \mathrm{~cm} . \omega$ is in units of $\mathrm{v}_{\mathrm{s}}^{2} / r_{\mathrm{g}} c$. Once the $\omega_{\max } \leq 1$ the non-resonant instability is not effective in amplifying the magnetic field.

In Fig. 1 we illustrate the influence of ion-neutral collisions for SNR parameters using a full numerical solution to the dispersion relation. Thermal damping is negligible, but a weak dependence on temperature enters via Eq. (8). We plot the maximum growth rate, as a function of ionisation fraction $x_{\mathrm{i}} \equiv n_{\mathrm{i}} / n$, taking the driving term $\zeta=0.01 x_{\mathrm{i}} \mathrm{v}_{\mathrm{s}} / c$ since the cosmic rays couple directly only to the ionised component. For higher density plasmas, a larger fraction of neutral particles is necessary to reduce the growth rate below the threshold value of $\mathrm{v}_{\mathrm{s}}^{2} / r_{\mathrm{g}} c$.

\section{Application to SNR}

We now investigate the role of the non-resonant instability in some typical supernova remants. Although it is beyond the scope of this work to estimate the possible saturated field strength from the non-resonant instability, we can nevertheless test the consistency of the analysis, by checking that the instability can enter the non-linear regime before being overtaken by the shock. Thus, the physical conditions far upstream $\left(B, n, x_{\mathrm{i}}, T\right)$ together with the shock 
speed $\mathrm{v}_{\mathrm{s}}$ and cosmic ray intensity $U_{\mathrm{cr}} / \rho_{\mathrm{i}} \mathrm{v}_{\mathrm{s}}{ }^{2}=0.1$, should combine to yield a growth rate in excess of $\mathrm{v}_{\mathrm{s}}{ }^{2} /\left(r_{\mathrm{g}} c\right)$. We investigate two extreme cases.

Firstly, for SN1006, the shock velocity is approximately $\mathrm{v}_{\mathrm{s}} \approx 2900 \mathrm{~km} \mathrm{~s}^{-1}$ the density is $0.05 \lesssim n \lesssim$ $0.25 \mathrm{~cm}^{-3}$, and the neutral fraction is small, $x_{\mathrm{i}} \approx 0.9$ [12]. Taking $n_{\mathrm{i}}=0.25 \mathrm{~cm}^{-3}$ gives $\zeta \mathrm{v}_{\mathrm{s}}{ }^{2} / \mathrm{v}_{\mathrm{A}}^{2} \sim 10$. The low density and shock speed, imply that the nonresonant mode is not very strongly driven, but the driving is nevertheless strong compared to the collision frequency. Very close to the shock, the growth time of the field is on the order of years, while further from the shock the growth rate decreases quite dramatically, as the number density of cosmic rays decreases. For the parameters given above the maximum growth rate is $\sim 10 \mathrm{v}_{\mathrm{s}}{ }^{2} / r_{\mathrm{g}} c$. The non-resonant mode may still amplify the field into the nonlinear regime.

The low neutral fraction in SN1006 means that ion-neutral collisions are not likely to dramatically reduce the instability. The interaction of a supernova blast wave with a molecular cloud, such as that observed close to the north-western rim of RX J1713.73946 would have a much more significant effect on the growth. Although the exact parameters are uncertain, molecular clouds are generally clumpy with interclump densities in the range $5-25 \mathrm{~cm}^{-3}$ and ionisation fractions not larger than $10 \%$ [13]. Adopting the values in [14] $T=10^{2} \mathrm{~K}, n=23 \mathrm{~cm}^{-3}, x_{\mathrm{i}}=$ $0.01, \mathrm{v}_{\mathrm{s}}=10^{8} \mathrm{~cm} \mathrm{~s}^{-1}$ the collision frequency is larger than the growth rate. The non-resonant driving term is unable to dominate over the Alfvén term and growth is driven by the cosmic rays themselves, at very long wavelengths $k r_{\mathrm{g}} \ll 1$. At shorter length scales, the waves are rapidly ion-neutral damped Alfvén waves. The shortest growth timescale is on the order of $\sim 10^{6}$ yrs. This value varies with choice of $r_{\mathrm{g}}$, temperature and density, but for high density and low ionisation fractions, the growth timescale is longer than the free expansion phase of a supernova remnant, or the lifetime of the wave in the shock precursor, suggesting that the Fermi acceleration mechanism may switch off upon interaction with a molecular cloud.

\section{Discussion}

It is now widely believed that magnetic field amplification is the best method for pushing the maximum energy beyond the Lagage-Cesarsky limit [1]. The non-resonant instability has been the most promising mechanism so far in that its growth rate is considerably faster than its resonant counterpart, and initial studies suggest that it does not saturate at a level $\delta B \lesssim B_{0}$. However, since the mechanism is non-resonant, with the fastest growing modes at very short wavelengths, the amplified field has very little effect on the acceleration of the maximum energy particles unless the energy is transferred to the large scale field by some non-linear process, as seen in some numerical simulations $[4,15]$. The results reported on in this paper extend the previous studies and allow application to more extreme environments. Such processes may be crucial for understanding particle acceleration at supernova remnant shocks in the galactic centre region, for example. Efficient acceleration is essential for this mechanism to occur. The largest uncertainty in our model arises in determining the value of the driving term $\zeta$. It is suggested, however, that efficient acceleration naturally leads to magnetic field amplification.

\section{Acknowledgments}

This research was jointly supported by COSMOGRID and the Max-Planck-Institut für Kernphysik, Heidelberg. The authors would like to thank the conference organisers.

\section{References}

[1] Lagage, P. O. and Cesarsky, C. J., A\&A 125, 249 (1983)

[2] Vink, J. and Laming, J. M. 2003, ApJ 584, 758 (2003)

[3] Völk, H. J. et al., A 8 A 433, 229 (2005)

[4] Bell, A. R., MNRAS 353, 550 (2004)

[5] Drury, L. O. C. et al., A $\mathscr{E} A$ 309, 1002 (1996)

[6] Kulsrud, R. and Pearce, W. P., ApJ 156, 445 (1969)

[7] Zweibel, E. G. and Shull, J. M., ApJ 259, 859 (1982)

[8] Bykov, A. M. and Toptygin, I. N. Astron. Lett. 31, 748 (2005)

[9] Reville, B. et al., A\&̊A 475, 435 (2007)

[10] Reville, B. et al., PPCF 48, 1741 (2006)

[11] Kulsrud, R. M. and Cesarsky, C. J. Astrophys. Lett. 8, 189 (1971)

[12] Raymond, J. C. et al., ApJ 659, 1257 (2007)

[13] Chevalier, R. A., ApJ 511, 798 (1999)

[14] Malkov, M. A. et al., ApJ 624, L37 (2005)

[15] Riquelme, M. and Spitkovsky, A., these proceedings 